\title{
A Novel Aerial Manipulator with Front Cutting Effector: Modeling, Control, and Evaluation
}

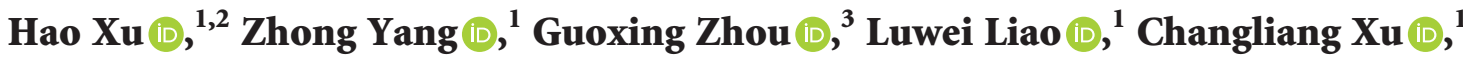 \\ Jiying Wu $\mathbb{D}^{1},{ }^{1}$ Qiuyan Zhang $\mathbb{D}^{4}{ }^{4}$ and Chi Zhang $\mathbb{D}^{1}$ \\ ${ }^{1}$ College of Automation Engineering, Nanjing University of Aeronautics and Astronautics, Nanjing 211106, China \\ ${ }^{2}$ School of Mathematics \& Physics, Anhui University of Technology, Ma'anshan 243032, China \\ ${ }^{3}$ Research Institute of UAV, Nanjing University of Aeronautics and Astronautics, Nanjing 211106, China \\ ${ }^{4}$ Guizhou Electric Power Research Institute, Guizhou Power Grid Co., Ltd., Guiyang 550000, China
}

Correspondence should be addressed to Zhong Yang; yangzhong@nuaa.edu.cn

Received 26 June 2021; Accepted 2 August 2021; Published 16 August 2021

Academic Editor: Shaohui Wang

Copyright ( 12021 Hao Xu et al. This is an open access article distributed under the Creative Commons Attribution License, which permits unrestricted use, distribution, and reproduction in any medium, provided the original work is properly cited.

\begin{abstract}
This paper proposes a novel aerial manipulator with front cutting effector (AMFCE) to address the aerial physical interaction (APhI) problem. First, the system uncertainty and external disturbance during the system movement and contact operation are estimated by modeling the entire robot and contact position. Next, based on the established model, the nonlinear disturbance observer (NDO) is used to estimate and compensate the unknown external disturbance of the system and the uncertainty of the model parameters in real time. Then, the nonsingular terminal synovial membrane control method is used to suppress the part that is difficult to estimate. Finally, a controller which is suitable for the movement and operation of the entire system is designed. The controller's performance is verified through experiments, and the results show that the design, modeling, and control of the entire system can achieve the APhI.
\end{abstract}

\section{Introduction}

The aerial manipulator is a kind of aerial robot that carries special operating tools and interacts with the operating object in the air to complete specific tasks [1]. The contact between the aerial manipulator and the environment combines the maneuverability of traditional work tools with the advantages of aerial robots such as long-range, fast speed, good maneuverability, and vast working space, making the application of aerial robots in modern industries possible $[2,3]$.

When this type of robot performs contact operations, it can work alone or cooperatively $[4,5]$; the object interacting with the aerial robot can be either known (for example, additional load by ground operators) or completely unknown (for example, contact inspection on the surface of unknown equipment). According to the different control devices, aerial manipulators can be divided into clamping, manipulating, cable hanging, and others [6]. Their applicability to different application scenarios is different in design, modeling, and control.

Helicopters can be used as one of the flight platforms to cut trees. By carrying a single degree-of-freedom (DOF) gripper, the helicopter uses the aerial robot to grasp and recover objects, which realizes the contact and manipulation between the aerial robot and the target [7]. Multirotors can also be used as a platform for aerial contact operations. For example, the GRASP laboratory has conducted in-depth research on the control, formation, and coordination of multirotor drones. As a result, the four-rotor aerial robot with a clamping mechanism developed can cooperate to move objects and build structures and cooperate in ensemble music, and so on [8].

Aerial manipulators designed for industrial applications are mainly concentrated in different industrial fields, including wall inspection, cutting trees, structure assembly, and object grasping. Our work is for tree cutting, and several researchers have researched this issue. Molina et al. $[9,10]$ 
developed an aerial robot equipped with a suspension control device for tree barrier cleaning based on a multirotor. The aerial robot must first fly to the vicinity of the branches to be cut, use the suspension manipulator to hang the body on the trunk to be cut, and then turn on the knife and saw to perform cutting operations. Hirai et al. [11] analyzed tree barrier cleaning task requirements, designed an ingenious suspension and cutting device, and formulated a detailed operation process for tree barrier cleaning. The robot can effectively cut trees with a diameter of about $3 \mathrm{~cm}$. Aerial manipulators with cable suspension structures are also used in the power industry. Azami et al. [12] developed a suspended chain saw aerial robot based on an 8-rotor UAV against the threat of tree barriers near high-voltage power lines. The aerial robot can fly to the target tree barrier under the condition that the motor actuator has sufficient speed and torque and use the suspended chain saw mechanism to perform fast cutting operations.

Most controllers of aerial manipulators are based on linear methods, especially the classical time-domain control method represented by PID technology [13], modern control theory represented by the LQR method [14], and robust control method represented by $\mathrm{H}$ infinite control method [15], and so on. However, many researchers have realized that the stability control of air manipulators based on the PID or LQR method is an optimal control method based on modern control theory and has now been successfully applied to the control system of a variety of air manipulators [16-18].

Since the existing system is often nonlinear, and the linear control method needs to be based on the linear model, this will limit the performance improvement of the control system. In order to overcome the limitations of linear control methods, more and more researchers have begun to devote themselves to the research of nonlinear control methods. Among them, the methods applied to aerial robot systems mainly include sliding mode control $[19,20]$ (SMC), backstepping [21], model predictive control (MPC) [22, 23], active disturbance rejection control $[24,25]$ (ADRC), and intelligent control [26-28].

In the process of operation, because the actuator of the aerial manipulator is in contact with objects in the environment, there will be uncertainty in its mass and moment of inertia, and the movement of the actuator (grabbing, cutting, etc.) will also bring unknown disturbances to the body. Therefore, selecting the most suitable antidisturbance control method is necessary based on its system characteristics and disturbance factors. The linear control method represented by the PID control has sound control effects for linear systems and is widely used in engineering. However, the linear method's decoupling ability and anti-interference ability are poor, and the control performance depends on the parameter adjustment, and the control performance cannot adapt to the change of the model well. Intelligent control, such as neural network control, has good disturbance and uncertainty estimation and compensation capabilities, but the network training relies on many data samples and powerful calculation capabilities, and it is challenging to implement engineering. Although the backstepping method in nonlinear control is not sensitive to external disturbances, it requires an accurate model to ensure the algorithm's accuracy.

Similarly, although the active disturbance rejection control has good robustness, it has many parameters, and the parameter tuning is complicated. Compared with backstepping and ADRC methods, MPC has good robustness and disturbance suppression capabilities, but it relies on repetitive online calculations, which requires high calculation performance of the control system, and it is not easy to implement. The SMC method changes the internal feedback control structure of the system so that the system state slides on the sliding mode surface and finally reaches a balance point. As a result, it has strong robustness to parameter uncertainty, model errors, and external interference. At the same time, considering that the traditional linear SMC cannot make the state variables converge in a finite time, this paper uses a nonsingular terminal sliding mode control (TSMC) to achieve convergence in time. In terms of disturbance suppression, most mentioned controllers rely on a priori known uncertainty upper bounds and had little knowledge about the system's unmodeled dynamics and unknown external disturbances. To solve this problem, we introduce disturbance observer (DO) into the design of the robot's position and attitude controller and use DO to estimate and compensate for unknown external disturbances and model parameter uncertainties in real time; the robustness of the nonsingular TSMC is used to suppress, to improve the disturbance suppression capability and robustness.

\section{Mathematical Model}

2.1. Coordinates and Conventions. First, we define the coordinate system representation, as shown in Figure 1.

The definition of each frame conforms to the right-hand rule. The earth frame $E\left\{O_{E}, X_{E}, Y_{E}, Z_{E}\right\}$ takes the earth's core as the coordinate origin $O_{E}$, and the $Z_{E}$-axis is perpendicular to the ground and points downward. The body frame $B\left\{O_{B}, X_{B}, Y_{B}, Z_{B}\right\}$ is fixed to the body, and its origin is at the center of mass (COM) of the aerial manipulator, and the $X_{B}$-axis points to the nose direction in the symmetry plane of the aerial robot. The $Z_{B}$-axis is perpendicular to the $X_{B}$-axis down in the plane of symmetry of the aerial robot, and then the $Y_{B}$-axis is determined according to the righthand rule.

The rotation matrix represents the rotation relationship from the earth frame to the body frame $R_{E}^{B}$ so that 


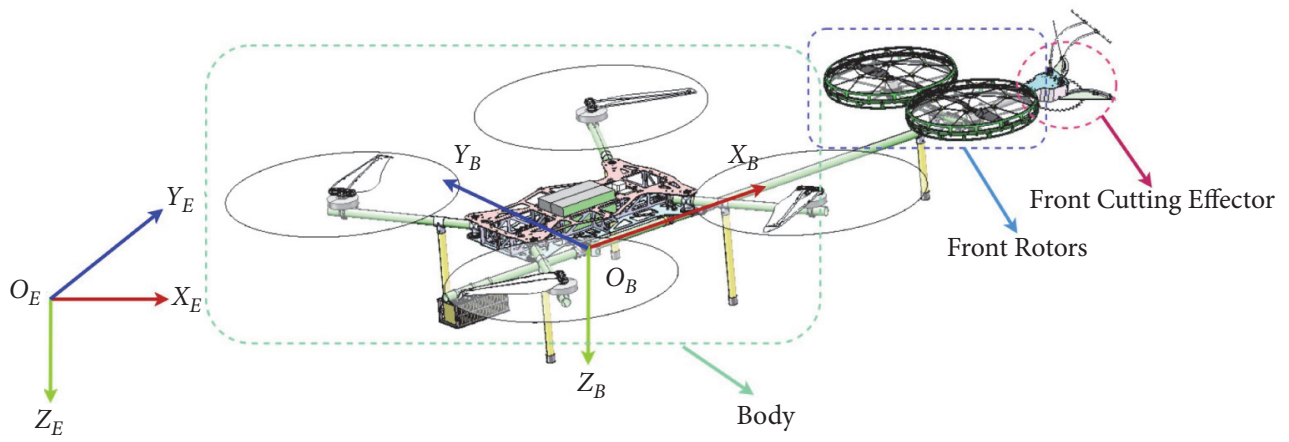

Figure 1: CAD model and frames of the AMFCE.

$$
R_{E}^{B}=\left[\begin{array}{ccc}
\cos \theta \cos \psi & \cos \theta \sin \psi & -\sin \theta \\
\sin \phi \sin \theta \cos \psi-\cos \phi \sin \psi & \sin \phi \sin \theta \sin \psi+\cos \phi \cos \psi & \sin \phi \cos \theta \\
\cos \phi \sin \theta \cos \psi+\sin \phi \sin \psi & \cos \phi \sin \theta \sin \psi-\sin \phi \cos \psi & \cos \phi \cos \theta
\end{array}\right]
$$

where $R_{E}^{B} \in S O(3), \quad S O(3) \triangleq\left\{\mathbf{R} \mid \mathbf{R}^{T} \mathbf{R}=\mathbf{I}_{3}, \operatorname{det}(\mathbf{R})=1, \mathbf{R} \in\right.$ $\left.\mathbb{R}^{3 \times 3}\right\}$.

2.2. Assumptions. The assumptions involved in the article are as follows:

(1) The whole system is regarded as a rigid body, and there is no flexible body

(2) When cutting, the body will not deform significantly

(3) When the FCE is working, it is controlled by a separate controller and has nothing to do with the motion controller of the entire system

2.3. Kinematic Modeling. According to Newton's law [4], the kinematics model of aerial robot can be expressed as

$$
\left\{\begin{array}{l}
\dot{P}=\mathbf{v}, \\
\dot{v}=\mathbf{g}+\mathbf{R}_{B}^{E} \frac{\mathbf{F}+\mathbf{F}_{h}}{m} .
\end{array}\right.
$$

In the earth frame, $\mathbf{P}=\left[\begin{array}{lll}x & y & z\end{array}\right]^{T}, \mathbf{v}=\left[\begin{array}{lll}v_{x} & v_{y} & v_{z}\end{array}\right]^{T}$, and $\mathbf{g}=\left[\begin{array}{lll}0 & 0 & g\end{array}\right]^{T}$ represent the position, velocity, and gravitational acceleration vector, respectively. $m$ represents the mass, $\mathbf{R}_{B}^{E}$ denotes the rotation matrix from the body frame to the earth's frame, which can be defined as

$$
\mathbf{R}_{B}^{E}=\left(\mathbf{R}_{E}^{B}\right)^{T} .
$$

We use $\mathbf{F}$ the total lift vector generated by the four main rotors in the body frame and the lift vector generated by the two front rotors in the body frame, which is denoted as

$$
\begin{gathered}
\mathbf{F}=\left[\begin{array}{lll}
0 & 0 & -f
\end{array}\right]^{T}, \quad f>0, \\
\left\{\begin{array}{l}
\mathbf{F}_{h}=\left[\begin{array}{lll}
0 & 0 & -f_{h}
\end{array}\right]^{T}, \\
f_{h}=f_{h l}+f_{h r}, \quad f_{h l}>0, f_{h r}>0,
\end{array}\right.
\end{gathered}
$$

where $f$ represents the total lift generated by the four rotors in the body frame. Moreover, $f_{h r}$ represents the lift provided by the left and suitable front rotors under the body frame. We can get

$$
\left\{\begin{array}{l}
\ddot{x}=\frac{\left[\cos \theta \cos \psi\left(f_{h l}+f_{h r}\right)-f(\cos \phi \sin \theta \cos \psi+\sin \phi \sin \psi)\right]}{m}, \\
\ddot{y}=\frac{\left[\cos \theta \sin \psi\left(f_{h l}+f_{h r}\right)-f(\cos \phi \sin \theta \sin \psi-\sin \phi \cos \psi)\right]}{m}, \\
\ddot{z}=g-\frac{\left[\sin \theta\left(f_{h l}+f_{h r}\right)+f(\cos \phi \cos \theta)\right]}{m} .
\end{array}\right.
$$


2.4. Dynamic Modeling. According to Newton's Euler equations [4], the dynamic model is expressed as

$$
\left\{\begin{array}{l}
\dot{\Theta}=\mathbf{R} \Omega, \\
\mathbf{J} \dot{\Omega}=-\Omega \times \mathbf{J} \mathbf{\Omega}+\mathbf{U}+\mathbf{D},
\end{array}\right.
$$

where $\Theta=\left[\begin{array}{lll}\phi & \theta & \psi\end{array}\right]^{T}$ and $\Omega=\left[\begin{array}{lll}p & q & r\end{array}\right]^{T}$, respectively, denote the Euler angle and the body angular velocity. The moment of inertia is expressed as $\mathbf{J}=\operatorname{diag}\left(J_{x}, J_{y}, J_{z}\right)$. D represents the model uncertain part and the external disturbance during flight and $\mathbf{U}=\left[\begin{array}{lll}U_{x} & U_{y} & U_{z}\end{array}\right]^{T}$ represents the total control torque on the attitude channel. $R$ represents the rotation matrix between the Euler angle change rate and the body angular velocity; the form is as follows:

$$
\mathbf{R}=\left[\begin{array}{ccc}
1 & \tan \theta \sin \phi & \tan \theta \cos \phi \\
0 & \cos \phi & -\sin \phi \\
0 & \frac{\sin \phi}{\cos \theta} & \frac{\cos \phi}{\cos \theta}
\end{array}\right] .
$$

Because the aerial manipulator we study moves slowly and has a low attitude change rate, $\mathbf{R}$ can be approximated as an identity matrix. So equation (7) can be simplified as

$$
\left\{\begin{array}{l}
\mathbf{J} \\
\mathbf{U}=-\mathbf{\Omega} \times \mathbf{J} \boldsymbol{\Omega}+\mathbf{U}+\mathbf{D}, \\
\mathbf{U}_{h},
\end{array}\right.
$$

where $\mathbf{U}_{c}=\left[\begin{array}{lll}U_{c x} & U_{c y} & U_{c z}\end{array}\right]^{T}$ represents the control torque of the attitude channel generated by the main rotor, $\mathbf{U}_{h}=$ $\left[\begin{array}{lll}U_{h x} & U_{h y} & U_{h z}\end{array}\right]^{T}$ is the control torque of the front rotors, and $\mathbf{D}=\left[\begin{array}{lll}D_{x} & D_{y} & D_{z}\end{array}\right]^{T}$ is the interference torque of the attitude channel. Therefore, the dynamic model can be expressed as follows:

$$
\left\{\begin{array}{l}
\ddot{\phi}=\dot{\theta} \dot{\psi} \frac{J_{y}-J_{z}}{J_{x}}+\frac{U_{c x}+U_{h x}}{J_{x}}+D_{x}, \\
\ddot{\theta}=\dot{\phi} \dot{\psi} \frac{J_{z}-J_{x}}{J_{y}}+\frac{U_{c y}+U_{h y}}{J_{y}}+D_{y} \\
\ddot{\psi}=\dot{\phi} \dot{\theta} \frac{J_{x}-J_{y}}{J_{z}}+\frac{U_{c z}+U_{h z}}{J_{z}}+D_{z} .
\end{array}\right.
$$

Let $\mathbf{P}_{h l}=\left[\begin{array}{lll}0 & -y_{l} & 0\end{array}\right]^{T}$ and $\mathbf{P}_{h r}=\left[\begin{array}{lll}0 & y_{r} & 0\end{array}\right]^{T}$ denote the installation positions of the two front rotors under the body frame, respectively. Then,

$$
\left\{\begin{array}{l}
U_{h x}=0, \\
U_{h y}=0, \\
U_{h z}=y_{l} f_{h l}-y_{r} f_{h r} .
\end{array}\right.
$$

Combining equations (5) and (11), we may get

$$
\left[\begin{array}{c}
F_{h} \\
U_{h z}
\end{array}\right]=\left[\begin{array}{cc}
1 & 1 \\
y_{l} & -y_{r}
\end{array}\right]\left[\begin{array}{l}
f_{h l} \\
f_{h r}
\end{array}\right], \quad f_{h l}>0, f_{h r}>0,
$$

and equation (12) represents the control allocation matrix of the front rotor.
The total lift generated by the four main rotors is given by

$$
f=C_{T}\left(\omega_{1}^{2}+\omega_{2}^{2}+\omega_{3}^{2}+\omega_{4}^{2}\right)
$$

Similarly, the torque generated by the four main rotors is as follows:

$$
\left\{\begin{array}{l}
U_{c x}=d C_{T}\left(-\frac{\sqrt{2} \omega_{1}^{2}}{2}-\frac{\sqrt{2} \omega_{2}^{2}}{2}+\frac{\sqrt{2} \omega_{3}^{2}}{2}+\frac{\sqrt{2} \omega_{4}^{2}}{2}\right), \\
U_{c y}=d C_{T}\left(\frac{\sqrt{2} \omega_{1}^{2}}{2}-\frac{\sqrt{2} \omega_{2}^{2}}{2}-\frac{\sqrt{2} \omega_{3}^{2}}{2}+\frac{\sqrt{2} \omega_{4}^{2}}{2}\right), \\
U_{c z}=C_{M}\left(\omega_{1}^{2}-\omega_{2}^{2}+\omega_{3}^{2}-\omega_{4}^{2}\right),
\end{array}\right.
$$

where $C_{T}$ is the lift coefficient and $\omega_{i}(i=1,2,3,4)$ represents the speed of the four rotors. $d$ denotes the distance from the center of the motor to the center of the body and $C_{M}$ is the antitorque coefficient of the motor.

According to equation (14), the control distribution relationship can be obtained as follows:

$$
M=A_{M} W
$$

where $M=\left[\begin{array}{llll}f & U_{c x} & U_{c y} & U_{c z}\end{array}\right]^{T}$ represents the driving force and torque generated by the rotors, the square of the rotation speed of each rotor is expressed as $W=\left[\begin{array}{llll}\omega_{1}^{2} & \omega_{2}^{2} & \omega_{3}^{2} & \omega_{4}^{2}\end{array}\right]^{T}$, and $A_{M}$ is the control allocation matrix:

$$
A_{M}=\left[\begin{array}{cccc}
C_{T} & C_{T} & C_{T} & C_{T} \\
-d C_{T} \frac{\sqrt{2}}{2} & -d C_{T} \frac{\sqrt{2}}{2} & d C_{T} \frac{\sqrt{2}}{2} & d C_{T} \frac{\sqrt{2}}{2} \\
d C_{T} \frac{\sqrt{2}}{2} & -d C_{T} \frac{\sqrt{2}}{2} & -d C_{T} \frac{\sqrt{2}}{2} & d C_{T} \frac{\sqrt{2}}{2} \\
C_{M} & -C_{M} & C_{M} & -C_{M}
\end{array}\right] .
$$

According to equation (9), it can be seen that both the front rotor and the main rotor participate in the attitude control, and the desired control torque generated by the attitude controller must be distributed to these two actuators. The relationship between the control torque produced by the main rotor and the control torque produced by the front rotor can be determined as follows:

$$
\left\{\begin{array}{l}
U_{z}=U_{c z}+U_{h z} \\
U_{c z}=\gamma U_{z}, \quad \gamma \in(0,1),
\end{array}\right.
$$

where $\gamma$ represents the ratio of the torque generated by the main rotor in the total torque. So far, according to equations (12), (16), and (17), the desired control torque generated by the attitude controller and the desired control force generated by the position controller can be converted into the rotational speed signals of the four main rotors and the two front rotors. To control the attitude and position of the aerial robot, the control allocation is expressed as follows: 


$$
\left[\begin{array}{l}
F_{t} \\
F_{h} \\
U_{x} \\
U_{y} \\
U_{z}
\end{array}\right]=\left[\begin{array}{cccccc}
C_{T} & C_{T} & C_{T} & C_{T} & 0 & 0 \\
0 & 0 & 0 & 0 & C_{T, h} & C_{T, h} \\
-C_{F} & -C_{F} & C_{F} & C_{F} & 0 & 0 \\
C_{F} & -C_{F} & -C_{F} & C_{F} & 0 & 0 \\
\gamma C_{M} & -\gamma C_{M} & \gamma C_{M} & -\gamma C_{M} & (1-\gamma) C_{T, h} y_{l} & -(1-\gamma) C_{T, h} y_{r}
\end{array}\right]\left[\begin{array}{c}
\omega_{1}^{2} \\
\omega_{2}^{2} \\
\omega_{3}^{2} \\
\omega_{4}^{2} \\
\omega_{5}^{2} \\
\omega_{6}^{2}
\end{array}\right]
$$

where $C_{F}=\sqrt{2} d C_{T} / 2$.

2.5. Environmental Modeling. In the actual flight environment, the aerial manipulator will be affected by various disturbances, so it is necessary to study the controller's performance under disturbances. This section mainly models the disturbances that may be involved in actual motion.

Considering the dynamic equation in the presence of gyro torque, equation (9) is rewritten as

$$
\mathrm{J} \Theta=-\boldsymbol{\Omega} \times \mathbf{J} \boldsymbol{\Omega}+\mathbf{G}_{a}+\mathrm{U}+\mathbf{D},
$$

and the gyro torque $\mathbf{G}_{a}$ can be expressed as follows:

$$
\mathbf{G}_{a}=\mathbf{J}_{R P} \boldsymbol{\Omega}_{r} \times \boldsymbol{\omega}_{r},
$$

where $\Omega_{r}$ is the motor angular velocity vector and $\omega_{r}$ is the rotation angular velocity of the motor shaft. Then the rotating component $k$ includes the motor-rotor system and the motor-saw system. The generated gyro torque is defined as

$$
\mathbf{G}_{a, k}=J_{R P, k} \Omega_{r, k} \mathbf{b}_{3} \times \boldsymbol{\omega}_{r},
$$

where $\mathbf{b}_{3}$ is the angular velocity direction of the rotating component and $J_{R P, k}$ is the total moment of inertia of the rotating component $k$. And there are

$$
\begin{aligned}
\mathbf{b}_{3} \times \boldsymbol{\omega}_{r} & =\mathbf{b}_{3}^{\times} \boldsymbol{\omega}_{r}=-\boldsymbol{\omega}_{r}^{\times} \mathbf{b}_{3}, \\
\boldsymbol{\omega}_{r} & =\left[\begin{array}{l}
\omega_{x} \\
\omega_{y} \\
\omega_{z}
\end{array}\right] \Longleftrightarrow \boldsymbol{\omega}_{r}^{\times}=\left[\begin{array}{ccc}
0 & -\omega_{z} & \omega_{y} \\
\omega_{z} & 0 & -\omega_{x} \\
-\omega_{y} & \omega_{x} & 0
\end{array}\right] .
\end{aligned}
$$

In summary, the gyro torque generated by the rotating component is as follows:

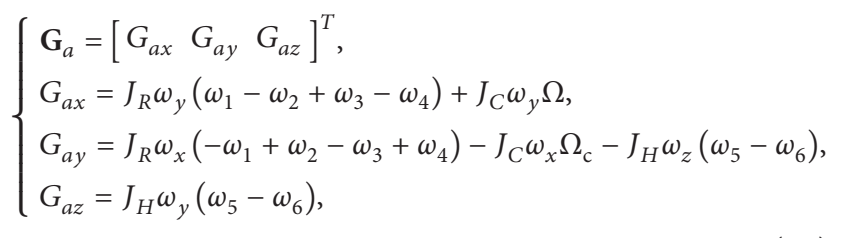

where $\Omega_{\mathrm{c}}$ is the rotational angular velocity of the saw, $J_{R}$ represents the moment of inertia of the rotor assembly, $J_{C}$ denotes the moment of inertia of the saw system, and $J_{H}$ represents the moment of inertia of the duct system.
The reverse torque generated by the cutting system is different from that by the rotor system described in equation (14).

When ignoring the air resistance encountered during the rotation of the saw, the motor-saw system can be equivalent to a fixed-axis spinning top, which can be obtained from the dynamic equation of the rotating rigid body:

$$
\mathbf{J}_{C} \dot{\beta}=\mathbf{T}_{R}-\mathbf{T}_{0} \text {, }
$$

where $\dot{\beta}$ represents the angular acceleration vector of the saw blade, $\mathbf{T}_{R}$ represents the motor drive torque vector, and $\mathbf{T}_{0}$ represents the load torque vector of the motor in the idling state of the saw blade. Then the reverse torque acting on the body which is caused by the rotation of the saw can be expressed as follows:

$$
\boldsymbol{\tau}_{r}=-\mathbf{T}_{R}=-\mathbf{J}_{C} \dot{\beta}+\mathbf{T}_{0}=\left[\begin{array}{lll}
0 & 0 & J_{C} \dot{\beta}+T_{0}
\end{array}\right]^{T} .
$$

2.6. Cutting Process Modeling. The force during the cutting process is shown in Figure 2.

It can be seen from Figure 2 that $F_{x}$ represents the main cutting force and $F_{y}$ represents the reaction force of the object to be cut on the aerial robot. Since $F_{y}$ passes through the COM of the robot, it does not affect the attitude channel, so only the disturbance caused by the main cutting force $F_{x}$ is considered.

The interference torque $\tau_{c}$ generated by the main cutting force on the robot is as follows:

$$
\boldsymbol{\tau}_{c}=F_{x} l_{s} \mathbf{b}_{3}
$$

where $\mathbf{b}_{3}=\left[\begin{array}{lll}0 & 0 & 1\end{array}\right]^{T}$ and $l_{s}$ is the distance from the front end of the saw to the COM of the aerial robot. Furthermore,

$$
\boldsymbol{\tau}_{\mathrm{c}}=\left[\begin{array}{lll}
0 & 0 & F_{x} l_{s}
\end{array}\right]^{T},
$$

and $F_{x}$ can be calculated as follows:

$$
F_{x}=\frac{K V_{0}}{v}=\frac{P_{c}}{v},
$$

where $V_{0}$ is the volume of branches cut by the saw blade per unit time and $P_{c}$ is the cutting power. $K$ and $v$ are the cutting force and linear speed of the saw, respectively. We have

$$
P_{e}=P_{c}+P_{0}
$$




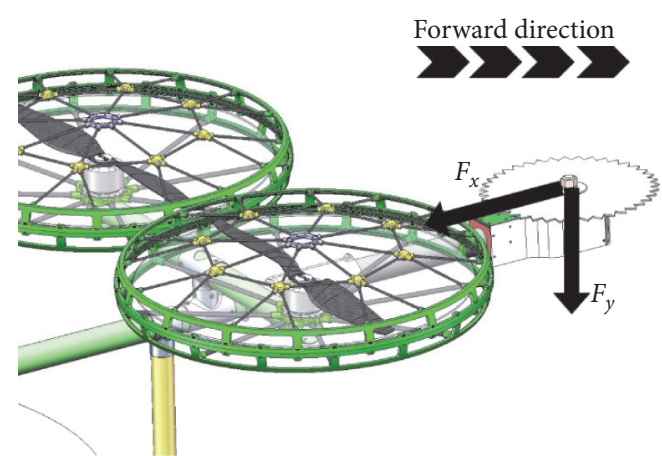

FIgURE 2: The direction of the force during cutting.

where $P_{0}$ and $P_{e}$, respectively, represent the no-load power and electromagnetic power of the DC motor.

When cutting objects in the environment, the main rotor keeps the body hovering. Take cutting a tree as an example, as shown in Figure 3.

During the cutting operation, FCE is in contact with the tree. The contact is equivalent to imposing external constraints on the entire robot, and the original dynamic model will change at this time. If the attitude controller is sensitive to model parameter changes, it will inevitably cause the aerial robot to lose stability or even crash. Therefore, it is necessary to establish a dynamic model of the aerial robot in the contact work environment to verify the robustness of the designed attitude controller. Furthermore, in the cutting process, we add the following assumptions:

(1) Ignore the influence of the contact object on the body during the cutting process

(2) The attitude of the aerial robot only changes in a small range during the cutting process

Assumption (1) makes contact between the aerial robot and the tree approximate to rigid body contact, simplifying the subsequent analysis. Assumption (2) ensures that the aerial robot's attitude changes at a slight angle during the cutting process because, in the actual test, the attitude angle changes too much during the cutting process, which will cause the cutting device to jam and even cause the aerial robot to lose control. Therefore, in the cutting process, the connection between the body and the tree can be equivalent to a universal joint, and the attitude rotation center of the aerial robot is transferred from the COM of the machine to the contact point $O_{C}$ of the saw and the tree. Use the contact point as the origin to establish a new frame system $C\left\{O_{C}, X_{C}, Y_{C}, Z_{C}\right\}$ so that the angle of the aerial robot body rotating around the $X_{C}$-axis is the roll angle, the angle rotating around the $Y_{C}$-axis is the pitch angle, and the angle rotating around the $Z_{C}$-axis is the yaw angle. Compared with equation (10), the change of the rotation center of the robot body changes the moment of inertia of the robot body, and equation (10) can be rewritten as follows:

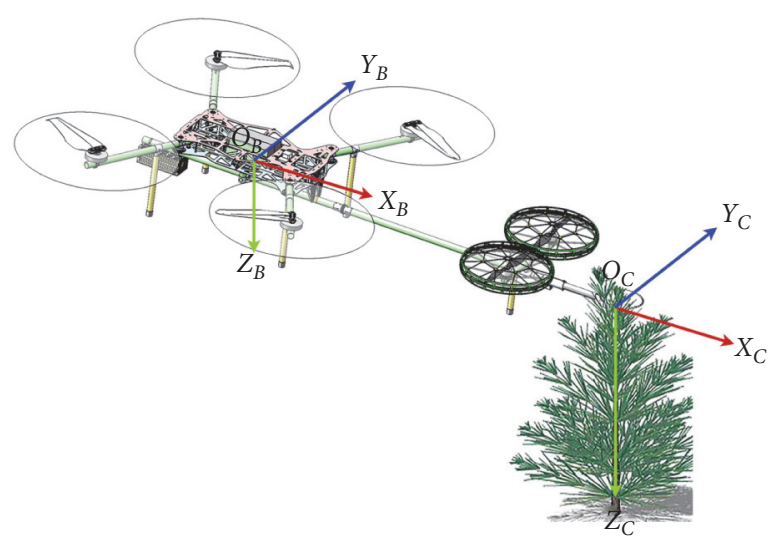

FIgURE 3: The cutting diagram.

$$
\left\{\begin{array}{l}
\ddot{\phi}=\dot{\theta} \dot{\psi} \frac{J_{y}^{\prime}-J_{z}^{\prime}}{J_{x}^{\prime}}+\frac{U_{c x}+U_{h x}}{J_{x}^{\prime}}+D_{x} \\
\ddot{\theta}=\dot{\phi} \dot{\psi} \frac{J_{z}^{\prime}-J_{x}^{\prime}}{J_{y}^{\prime}}+\frac{U_{c y}+U_{h y}}{J_{y}^{\prime}}+D_{y} \\
\ddot{\psi}=\dot{\phi} \dot{\theta} \frac{J_{x}^{\prime}-J_{y}^{\prime}}{J_{z}^{\prime}}+\frac{U_{c z}+U_{h z}}{J_{z}^{\prime}}+D_{z}
\end{array}\right.
$$

with $\left(J_{x}^{\prime}, J_{y}^{\prime}, J_{z}^{\prime}\right)$ being the new moment of inertia of the body. Therefore, the control allocation matrix of the main rotor of the aerial robot has also changed to

$$
A_{M}=\left[\begin{array}{cccc}
C_{T} & C_{T} & C_{T} & C_{T} \\
-d C_{T} \frac{\sqrt{2}}{2} & -d C_{T} \frac{\sqrt{2}}{2} & d C_{T} \frac{\sqrt{2}}{2} & d C_{T} \frac{\sqrt{2}}{2} \\
-l C_{T} & -(l+\sqrt{2} d) C_{T} & -(l+\sqrt{2} d) C_{T} & -l C_{T} \\
C_{M} & -C_{M} & C_{M} & -C_{M}
\end{array}\right],
$$

where $l$ is the distance from the line between the centers of rotors 1 and 4 to the contact point $O_{C}$. Compared with equation (16), the rotation center change of the aerial robot body changes the distance from the main rotor to the rotation axis, thereby changing the control allocation matrix of the attitude channel. In summary, the robot controller needs to have good robustness and disturbance suppression ability to achieve stable robot control.

\section{Controller Design}

Considering external disturbances and model uncertainties in the system, the design of a control law with strong, robust 
antidisturbance ability is of great significance to improve the robot's adaptability to complex environments. This paper uses a sliding mode method with strong robustness and disturbance suppression to design the control law. At the same time, it discusses the basic principles of TSMC and NFTSMC with the finite-time convergence of SMC as the starting point. Then, based on the mathematical model of the system established above, the position and attitude controller of the robot is established using the NFTSM method. Finally, a nonlinear disturbance observer (NDO) is designed to observe the external disturbance of the system and compensate the controller, which improves the robustness of the control system.

3.1. Overview. The traditional SMC uses a linear sliding mode surface. When the system's state reaches the sliding mode surface, the system's response will become insensitive to the uncertain internal parameters of the system and external disturbances, which makes the SMC controller has good robustness. Nevertheless, the disadvantage of traditional SMC is that the convergence of the system's state to the equilibrium point is asymptotic and cannot achieve finite-time convergence. In recent years, the terminal sliding mode control (TSMC) has shown some excellent properties in applications, such as the system state error can converge to zero in a finite time, and it has higher steady-state accuracy than traditional SMC. However, when the general TSMC method is close to the system's equilibrium state, the controller's output will appear singular. In order to solve the above singular problems, the nonsingular fast terminal sliding mode (NSFTSM) method was proposed and applied to some systems with external disturbances and uncertain parameters.

There is a coupling relationship between the position and attitude of the robot. When realizing the position tracking control, only the yaw angle of the three attitude angles can be independently controlled, and the position control in the horizontal direction requires roll angle and pitch angle as the execution link of the positioning system. In particular, the robot in the cutting operation state can achieve horizontal forward flight because the assistance of the front rotor can make the forward position control no longer rely on the significant change of the pitch angle. However, the robot is still an underactuated system, with only five control variables in the controller.

First, the general control law adopts the control strategy of inner and outer loops and divides the six-state quantities into the inner loop with faster response speed and the outer loop with slower response speed. The inner loop is attitude control, and the outer loop is position control.

$$
\left[\begin{array}{c}
\omega_{1}^{2} \\
\omega_{2}^{2} \\
\omega_{3}^{2} \\
\omega_{4}^{2}
\end{array}\right]=\frac{1}{4}\left[\begin{array}{cccc}
1 & -1 & 1 & 1 \\
1 & -1 & -1 & -1 \\
1 & 1 & -1 & 1 \\
1 & 1 & 1 & -1
\end{array}\right] \operatorname{diag}\left(\frac{1}{C_{T}}, \frac{\sqrt{2}}{d C_{T}}, \frac{\sqrt{2}}{d C_{T}}, \frac{1}{C_{M}}\right)\left[\begin{array}{c}
F_{t} \\
U_{x} \\
U_{y} \\
U_{c z}
\end{array}\right],
$$

$\left[\begin{array}{l}\omega_{5}^{2} \\ \omega_{6}^{2}\end{array}\right]=\frac{C_{T, h}}{2}\left[\begin{array}{cc}1 & 1 \\ 1 & -1\end{array}\right] \operatorname{diag}\left(1, \frac{1}{y_{r}}\right)\left[\begin{array}{c}F_{h} \\ U_{h z}\end{array}\right]$,

where $\left(F_{t}, F_{h}\right)$ represents the desired total lift and the desired forward thrust. $\left(U_{x}, U_{y}, U_{z}\right)$ denote the desired control torque around the $x$-axis of the body frame $B\left\{O_{B}, X_{B}, Y_{B}, Z_{B}\right\}$. The position control input in the outer loop is the actual position $(x, y, z)$ and the desired position $\left(x_{\text {des }}, y_{\text {des }}, z_{\text {des }}\right)$, and the output is the desired value $\left(\phi_{\text {des }}, \theta_{\text {des }}, \psi_{\text {des }}\right)$ of the roll and pitch angles and the desired forward thrust $F_{h}$. The attitude control of the inner loop calculates the desired moment $\left(U_{x}, U_{y}, U_{z}\right)$ in the body frame according to the desired attitude angle $\left(\phi_{\text {des }}, \theta_{\text {des }}, \psi_{\text {des }}\right)$ and the actual attitude angle $(\phi, \theta, \psi)$.

We designed a dual closed-loop position and attitude controller. As shown in Figure 4, the control allocation is converting the control output calculated by the controller into the desired motor speed. Equations (32) and (33) describe the specific conversion relationship.

When used as an attitude tracking controller, the position control will not participate in the process, and the desired attitude $\left(\phi_{\text {ref }}, \theta_{\text {ref }}, \psi_{\text {ref }}\right)$ is externally given. $F_{h}$ is the same external setting, and $F_{t}$ is determined according to equation (6) combining $\phi, \theta$ and $z$-channel control variables. The following section will explain the control design of the inner loop and outer loop, respectively.

3.2. Attitude Control. This section will implement the design of the attitude controller based on the dynamic model established in Section 2. The problems we propose to solve by the attitude controller are as follows:

(1) When cutting different trees, it is necessary to carry different sawing equipment, which leads to uncertainty in the mass and moment of inertia of the robot

(2) The saw will produce interference force and interference torque on the body when it is working, which introduces disturbances to the attitude control

(3) During the operation, the contact between the robot and the tree will cause its own model to change, 


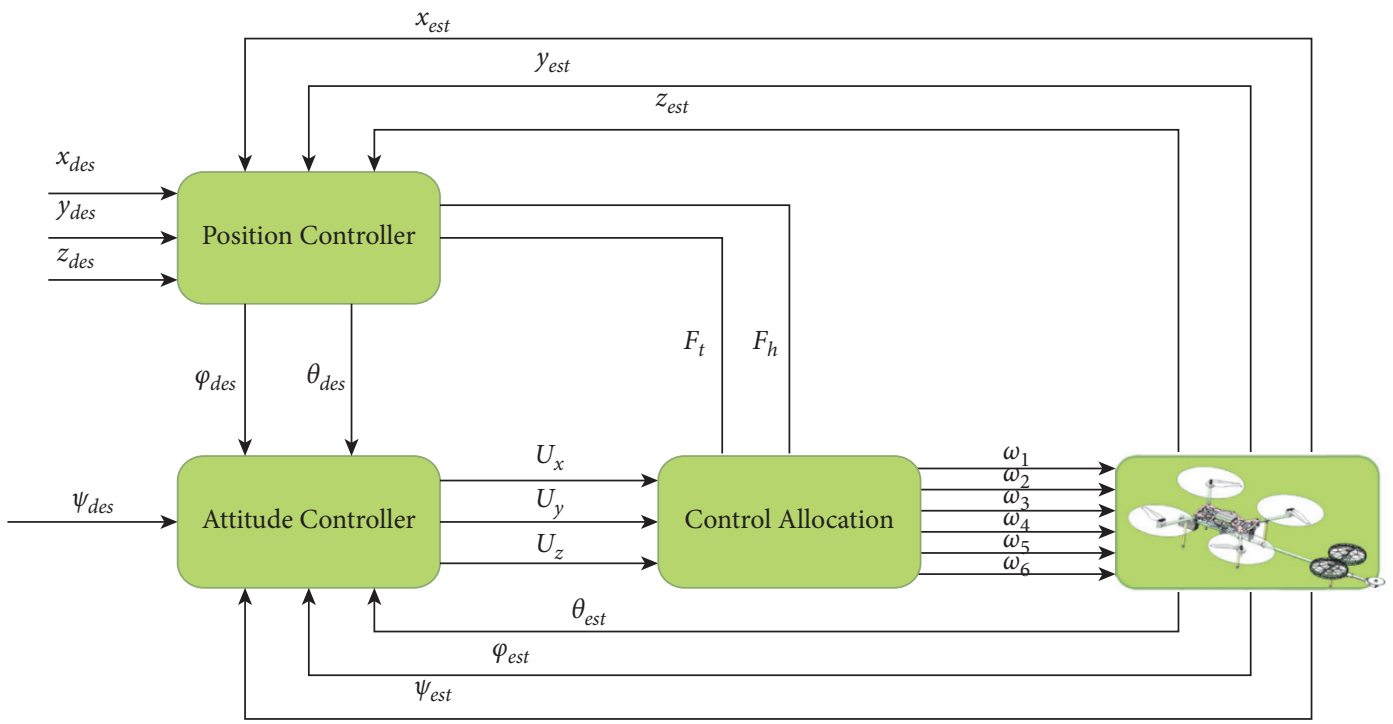

FIgURE 4: The control diagram.

which is mainly reflected in the change of the moment of inertia

Therefore, the robot has the uncertainty of mass and moment of inertia and unknown external disturbances in the attitude channel. Take the pitch channel as an example to rewrite the dynamic equation of the system, which is shown as equation (7), into the following form:

$$
\begin{aligned}
\left(J_{y}+\Delta J_{y}\right) \ddot{\theta} & =\dot{\phi} \dot{\psi}\left(J_{z}+\Delta J_{y}-J_{x}+\Delta J_{y}\right)+U_{y}+\left(J_{y}+\Delta J_{y}\right) D_{y}, \\
J_{y} \ddot{\theta} & =\dot{\phi} \dot{\psi}\left(J_{z}-J_{x}\right)+U_{y}+\dot{\phi} \dot{\psi}\left(\Delta J_{z}-\Delta J_{x}\right)+\left(J_{y}+\Delta J_{y}\right) D_{y}-\Delta J_{y} \ddot{\theta} \\
& =-G_{y}+T_{y}+d,
\end{aligned}
$$

where $d=\dot{\phi} \dot{\psi}\left(\Delta J_{z}-\Delta J_{x}\right)+\left(J_{y}+\Delta J_{y}\right) D_{y}-\Delta J_{y} \ddot{\theta}$ represents the total disturbance of the system. $T_{y}=U_{y}$ represents the control input of the attitude channel, and the coupling part in the attitude channel is expressed as $G_{y}=$ $-\dot{\phi} \dot{\psi}\left(J_{z}-J_{x}\right)$. We design a disturbance observer to observe the total disturbance $d$. The disturbance part observed by the disturbance observer is used to compensate for the controller output, and the unobserved disturbance part is suppressed by the robustness of the NFTSM controller itself. Figure 5 shows the structure diagram of the NDO-based NFTSM attitude controller.

Through $\dot{p}=L J_{y} \ddot{\theta}$, then we have $p=L J_{y} \dot{\theta}$. The NDO of the attitude channel can be set as follows:

$$
\left\{\begin{array}{l}
\hat{d}=z_{t}+L J_{y} \dot{\theta}, \\
\dot{z}_{t}=-L \widehat{d}+L\left(G_{y}-T_{y}\right) .
\end{array}\right.
$$

Let $\tilde{d}=\hat{d}-d$, then the stability of the above observer can be proved as follows:

$$
\begin{aligned}
\dot{\tilde{d}} & =\dot{\hat{d}}-\dot{d}=\dot{z}_{t}+L J_{y} \ddot{\theta}=\dot{z}_{t}+L\left(-G_{y}+T_{y}+d\right) \\
& =-L(\hat{d}-d)=-L \tilde{d},
\end{aligned}
$$

where $L$ is the bandwidth of the observer.

Next is the design of the NFTSM attitude controller. According to equation (35), we can get

$$
U_{y}=-J_{y}\left[-\frac{G_{y}}{J_{y}}+\frac{\hat{d}}{J_{y}}-\ddot{\theta}_{\text {ref }}+\frac{1}{\mu k_{2}}\left|e_{2}\right|^{2-k_{2}} \operatorname{sign}\left(e_{2}\right)\left(1+\eta k_{1}\left|e_{1}\right|^{k_{1}-1}\right)+k_{3} \operatorname{sign}(s)+k_{4} \operatorname{sig}^{\lambda} s\right] .
$$

So far, the design of the nonsingular terminal sliding mode controller based on NDO for the pitch channel is completed. The control law design of the roll and yaw channel is similar to the above process. Finally, the overall inner loop control law can be obtained by combining the attitude control laws of the three channels. 


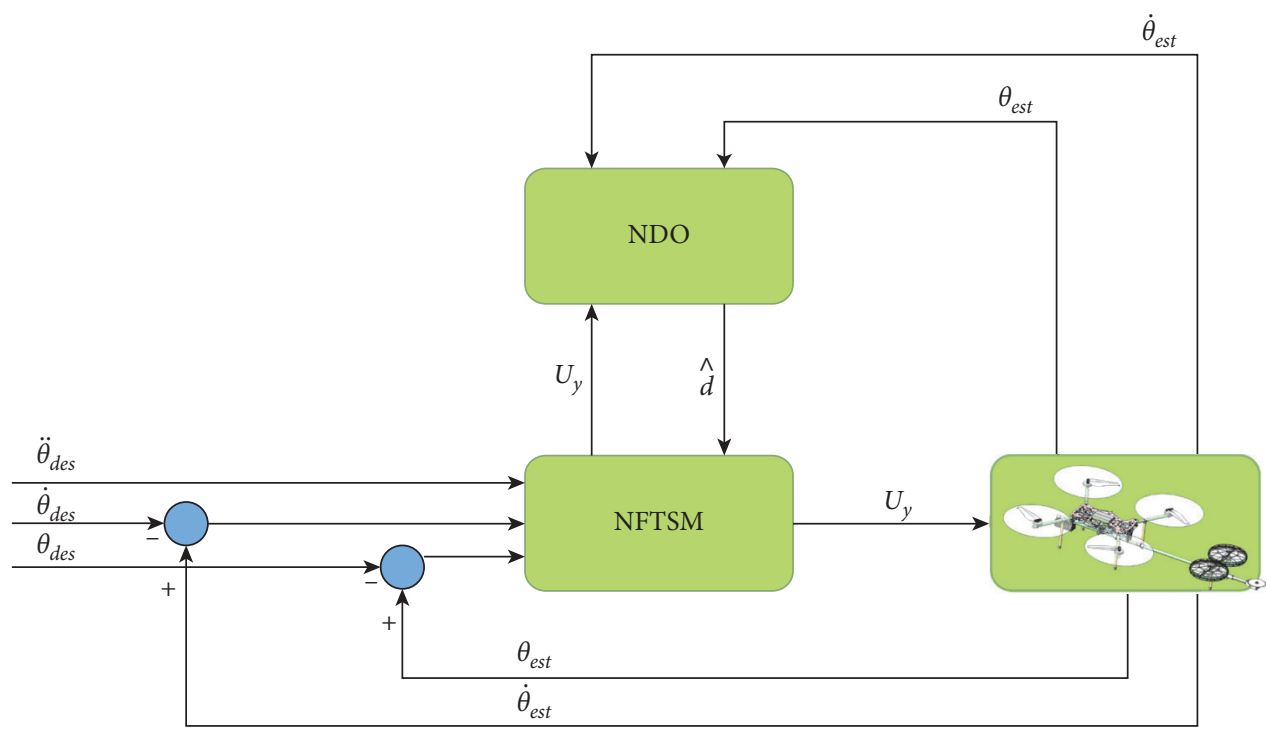

FIGURE 5: Control diagram of the pitch channel.

3.3. Position Control. The function to be realized by the position controller is to fly automatically according to a preset trajectory. First, we define a frame $K\left\{O_{K}\right.$, $\left.X_{K}, Y_{K}, Z_{K}\right\}$, the origin of the coordinate is at the COM of the Robot, and the $Z_{K}$-axis is perpendicular to the $X_{E} Y_{E}$-plane of the earth's fixed frame and points to the center of the earth. The $X_{K}$-axis coincides with the projection of the $X_{B}$-axis in the $X_{E} Y_{E}$ plane, and the $Y_{K}$-axis coincides with the projection of the body's $Y_{B}$-axis in the $X_{E} Y_{E}$ plane. In this frame, the horizontal acceleration generated by rolling is along the $Y_{K}$-axis direction and the horizontal acceleration generated by pitching is along the $X_{K}$-axis direction. Since the position information is described in the earth frame, it is necessary to decompose the horizontal position error and velocity error in the earth frame to the $X_{K}$-axis and $Y_{K}$-axis of the reference frame $K\left\{O_{K}, X_{K}, Y_{K}, Z_{K}\right\}$. The conversion matrix is shown in equation (39), where $\psi$ represents the yaw angle of AMFCE. Equation (40) represents the kinematic equation of AMFCE in the frame $K\left\{O_{K}, X_{K}, Y_{K}, Z_{K}\right\}$.

$$
\begin{aligned}
& \mathbf{R}_{E}^{K}=\left[\begin{array}{ccc}
\cos \psi & \sin \psi & 0 \\
-\sin \psi & \cos \psi & 0 \\
0 & 0 & 1
\end{array}\right], \\
& \left\{\ddot{x}_{r}=\cos \phi \sin \theta \frac{-F_{t}}{m}+\cos \theta F_{h}, \ddot{y}_{r}=-\sin \phi \frac{-F_{t}}{m}, \ddot{z}_{r}=g-(\cos \phi \cos \theta) \frac{F_{t}}{m}-\sin \theta F_{h} .\right.
\end{aligned}
$$

The following is the design of the height channel controller. Considering the unknown disturbance and the uncertainty of the AMFCE quality, the height $z$ model can be rewritten as follows:

$$
\begin{aligned}
(m+\Delta m) \ddot{z}_{r} & =(m+\Delta m) g-(m+\Delta m) \sin \theta F_{h}-\cos \phi \cos \theta F_{t}+D_{h}, \\
\frac{m}{\cos \phi \cos \theta} \ddot{z}_{r} & =\frac{m g}{\cos \phi \cos \theta}-F_{t}+\frac{\left(\Delta m g-\Delta m \ddot{z}_{r}-m \sin \theta F_{h}-\Delta m \sin \theta F_{h}\right)}{\cos \phi \cos \theta} \\
& =-G_{\text {alt }}+T_{\text {alt }}+d_{\text {alt }},
\end{aligned}
$$

where $\quad d_{\text {alt }}=\left(\Delta m g-\Delta m \ddot{z}_{r}-m \sin \theta F_{h}-\Delta m \sin \theta F_{h}\right) /$ $\cos \phi \cos \theta$ represents the total disturbance of the system and $T_{\text {alt }}=-F_{t}$ denotes the equivalent control variable. Therefore, the disturbance observer (DO) can be designed to observe the total disturbance $d$; the disturbance observed by the DO is used to compensate the controller's output, and the 
unobserved part is suppressed by the robustness of the NFTSM controller itself.

Then the NDO design of the height channel is carried out, so that $\dot{p}=L(m / \cos \theta \cos \phi) \ddot{z}_{r}$ has $p=L(\mathrm{~m} /$ $\cos \theta \cos \phi) \dot{z}_{r}$. According to equation (43), the NDO setting of the attitude channel is as follows:

$$
\left\{\begin{array}{l}
\widehat{d}_{\mathrm{alt}}=z_{t}+L \frac{m}{\cos \phi \cos \theta} \dot{z}_{r}, \\
\dot{z}_{t}=-L \widehat{d}_{\mathrm{alt}}+L\left(G_{\mathrm{alt}}-T_{\mathrm{alt}}\right) .
\end{array}\right.
$$

Next, the design of the NFTSM height controller is carried out. According to equation (42), we can get

$$
T_{\text {alt }}=-\frac{m}{\cos \phi \cos \theta}\left[g+\frac{\cos \phi \cos \theta}{m} \widehat{d}_{\mathrm{alt}}-\ddot{z}_{K, \mathrm{des}}+\frac{1}{\mu k_{2}}\left|e_{2}\right|^{2-k_{2}} \operatorname{sign}\left(e_{2}\right)\left(1+\eta k_{1}\left|e_{1}\right|^{k_{1}-1}\right)+k_{3} \operatorname{sign}(s)+k_{4} \operatorname{sig}^{\lambda} s\right] .
$$

So far, the design of the TSM controller with height channel is completed. The output of the horizontal channel is $\left(\phi_{\text {des }}, \theta_{\text {des }}, F_{h}\right)$, but according to the kinematic equation of AMFCE, these three control variables do not have a one-toone correspondence with the horizontal motion variables, which means there is a control coupling between the horizontal motion variables. The expression on the right side of equation (40) is replaced by $\left(u_{1}, u_{2}\right)$ as a whole; then,

$$
\left\{\begin{array}{l}
u_{1}=\cos \phi \sin \theta \frac{-F_{t}}{m}+\cos \theta F_{h}, \\
u_{2}=\sin \phi \frac{F_{t}}{m} .
\end{array}\right.
$$

The AMFCE studied in this paper has two working states: one is the cutting operation and the other is the free flight when not cutting. We have

$$
\left\{\begin{array}{l}
\phi_{\mathrm{des}}=\arcsin \left(\frac{m u_{2}}{F_{t}}\right), \\
\theta_{\mathrm{des}}=0, \\
F_{h}=\frac{\left(u_{1}+\left(\cos \phi \sin \theta F_{t} / m\right)\right)}{\cos \theta} .
\end{array}\right.
$$

AMFCE is in the cutting operation state; combine equation (40) to calculate the control output $\theta_{\text {des }}$, that is,

$$
\left\{\begin{array}{l}
\phi_{\mathrm{des}}=\arcsin \left(\frac{m u_{2}}{F_{t}}\right), \\
\theta_{\mathrm{des}}=-\arcsin \left(\frac{m u_{1}}{F_{t} \cos \phi}\right), \\
F_{h}=0 .
\end{array}\right.
$$

Based on equation (47), consider $\left(u_{1}, u_{2}\right)$ as the virtual control quantity introduced into the system, and put it into equation (40) to obtain

$$
\left\{\begin{array}{l}
\ddot{x}_{r}=u_{1} \\
\ddot{y}_{r}=u_{2}
\end{array}\right.
$$

The following takes the $X_{K}$-channel as an example to illustrate the design process of the horizontal channel controller. Assuming $z_{1}=x_{K}, z_{2}=\dot{x}_{K}$, equation (48) on the $X_{K}$-channel can be rewritten as follows:

$$
\left\{\begin{array}{l}
\dot{z}_{1}=z_{2} \\
\dot{z}_{2}=u_{1}+d_{1} \\
x_{K}=z_{1}
\end{array}\right.
$$

where $d_{1}$ is the total disturbance of the $X_{K}$-channel and $u_{1}$ is the virtual control quantity, and we can get

$$
\left\{\begin{array}{l}
\hat{d}_{1}=z_{t}+L \dot{z}_{1} \\
\dot{z}_{t}=-L\left(\widehat{d}_{1}+u_{1}\right)
\end{array}\right.
$$

According to equation (42),

$$
\begin{aligned}
u_{1}= & -\left[\hat{d}_{1}-\ddot{x}_{K, \mathrm{des}}+\frac{1}{\mu k_{2}}\left|e_{2}\right|^{2-k_{2}} \operatorname{sign}\left(e_{2}\right)\left(1+\eta k_{1}\left|e_{1}\right|^{k_{1}-1}\right)\right. \\
& \left.+k_{3} \operatorname{sign}(s)+k_{4} \operatorname{sig}^{\lambda} s\right] .
\end{aligned}
$$

The controller design of the $X_{K}$-channel is similar to that of the $Y_{K}$-channel. Therefore, we can get the virtual control quantity $\left(u_{1}, u_{2}\right)$ and the ducted thrust control quantity $F_{h}$. Combining equations (46) and (47) can obtain the control output $\left(\phi_{\mathrm{des}}, \theta_{\mathrm{des}}, F_{h}\right)$ of the horizontal channel in the outer loop controller. Then combine the position control laws of the three channels to get the outer loop control law of AMFCE.

\section{Evaluation}

4.1. System Description. We have realized the aerial robot with Figure 1 as the CAD model, and the actual object is shown in Figure 6.

Table 1 summarizes the physical parameters of the robot, and Table 2 shows the controller parameters. 


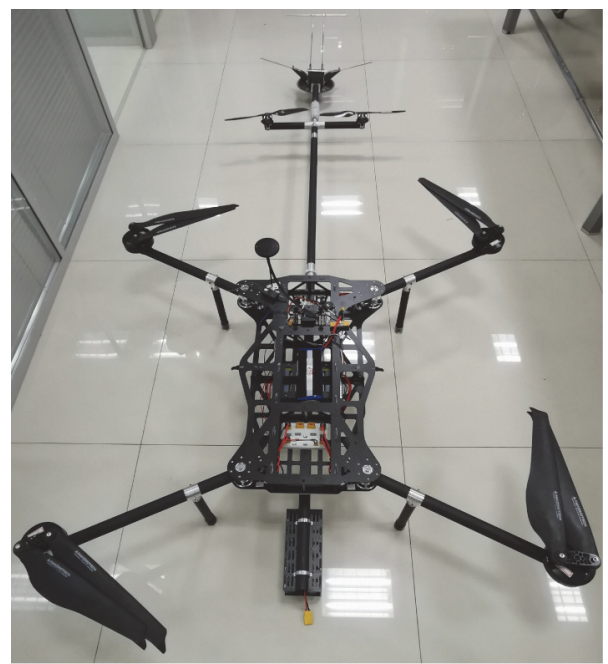

FIgURE 6: The final version of the AMFCE.

TABLe 1: Physical parameters.

\begin{tabular}{lcc}
\hline Parameters & Value & Unit \\
\hline AMFCE's mass, $m$ & 19.12 & $\mathrm{~kg}$ \\
Rotor arm length, $d$ & 1.37 & $\mathrm{~m}$ \\
Distance from front rotor to COM, $y_{l}$ & 1.54 & $\mathrm{~m}$ \\
Inertia tensor in the $x$-axis, $J_{x}$ & 0.79 & $\mathrm{~kg} \cdot \mathrm{m}^{2}$ \\
Inertia tensor in the $y$-axis, $J_{y}$ & 5.79 & $\mathrm{~kg} \cdot \mathrm{m}^{2}$ \\
Inertia tensor in the $z$-axis, $J_{z}$ & 5.47 & $\mathrm{~kg} \cdot \mathrm{m}^{2}$ \\
Motor time constant, $T_{r}$ & 0.064 & $\mathrm{~s}$ \\
Scale factor, $\gamma$ & 0.72 & - \\
Lift coefficient, $C_{T}$ & $1.435 \times 10^{-5}$ & $\mathrm{~N} \cdot \mathrm{rad}^{-2}$ \\
Antitorque coefficient, $C_{M}$ & $2.16 \times 10^{-7}$ & $\mathrm{~N} \cdot \mathrm{m}^{2} \cdot \mathrm{rad}^{-2}$ \\
\hline
\end{tabular}

TABLE 2: Controller parameters.

\begin{tabular}{|c|c|c|c|c|c|c|c|}
\hline & Parameters & $\phi$ & $\theta$ & $\psi$ & $x$ & $y$ & $z$ \\
\hline NDO & $L$ & 150 & 125 & 125 & 75 & 75 & 75 \\
\hline \multirow{7}{*}{ NFTSM } & $k_{1}$ & 20 & 26 & 35 & 21 & 24 & 30 \\
\hline & $k_{2}$ & 1.25 & 1.25 & 1.25 & 1.5 & 2.1 & 1.8 \\
\hline & $k_{3}$ & 5 & 2.5 & 2.5 & 5 & 2.5 & 2.5 \\
\hline & $k_{4}$ & 5 & 2.5 & 2.5 & 5 & 2.5 & 2.5 \\
\hline & $\mu$ & 0.15 & 0.1 & 0.12 & 0.32 & 0.2 & 0.2 \\
\hline & $\eta$ & 10 & 10 & 10 & 4.2 & 44 & 3.5 \\
\hline & $\lambda$ & 0.1 & 0.1 & 0.1 & 1.1 & 2.1 & 1.2 \\
\hline \multirow{3}{*}{ msat } & $\Delta$ & 0.3 & 0.3 & 0.3 & 0.5 & 0.5 & 0.5 \\
\hline & $\delta$ & 0.1 & 0.1 & 0.1 & 0.1 & 0.1 & 0.1 \\
\hline & $\alpha$ & 0.005 & 0.005 & 0.005 & 0.005 & 0.005 & 0.005 \\
\hline
\end{tabular}

4.2. Simulation. The controller was tested in simulation, which used Gazebo [29] as a physics environment and to model AMFCE. We also used the RotorS-plugin [30] to simulate the thrust motors. The CAD model of AMFCE was then loaded into this environment. Similar to the existing system, the commands were sent via the robot operating system to the controller.

The simulation and actual experiment tasks are the same to focus on the actual flight experiment in the next section.
4.3. Free-Flight Experiment. In order to verify the performance of the NDO-based NFTSM controller more thoroughly, this section will conduct the AMFCE trajectory tracking experiment.

We designed an experiment to make the robot fly around a tree. The purpose of this experiment is to make the robot fly around the cutting object. We chose a tree as the center of the circular trajectory on the experimental site and let the robot fly around the tree (Figure 7). 

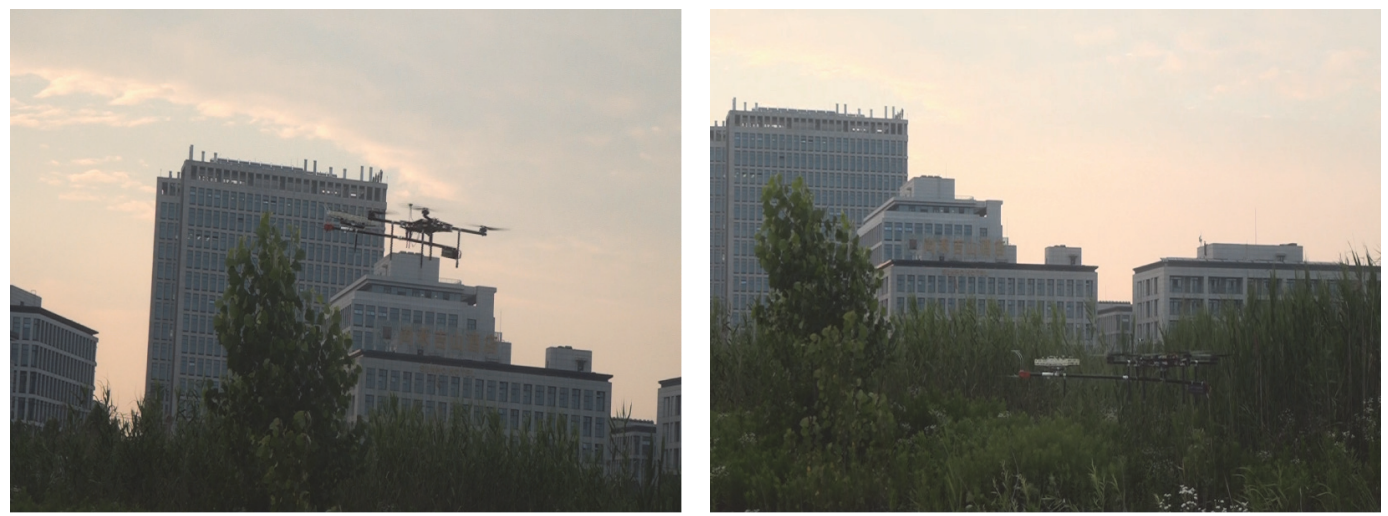

Figure 7: Snapshots of the free-flight experiment.

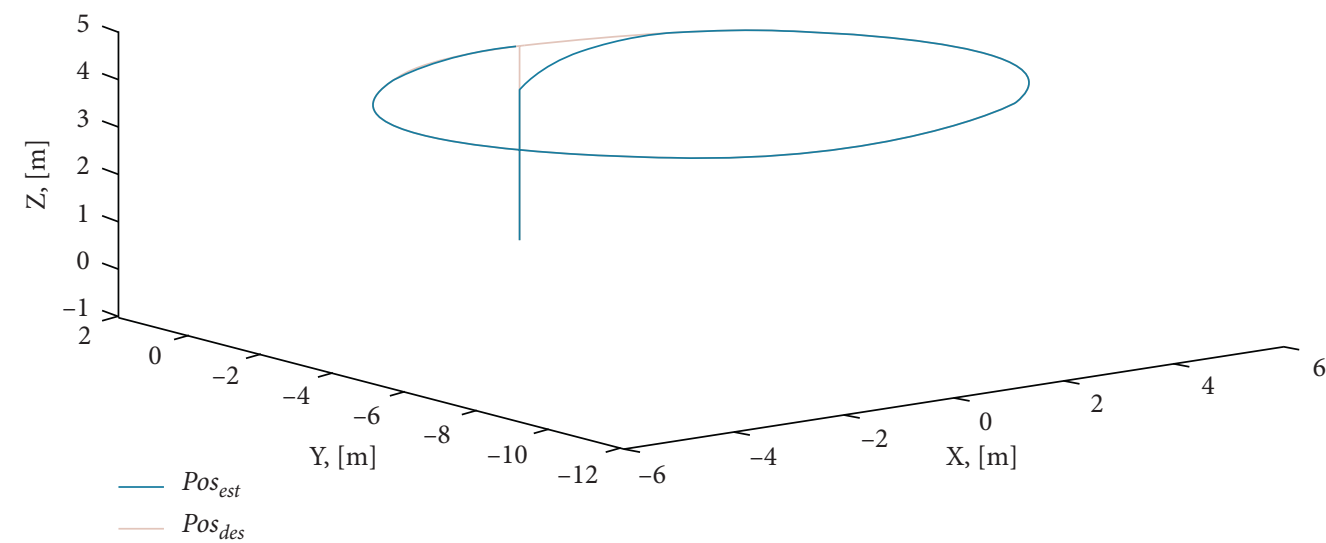

Figure 8: Tracking trajectory in the free-flight experiment.

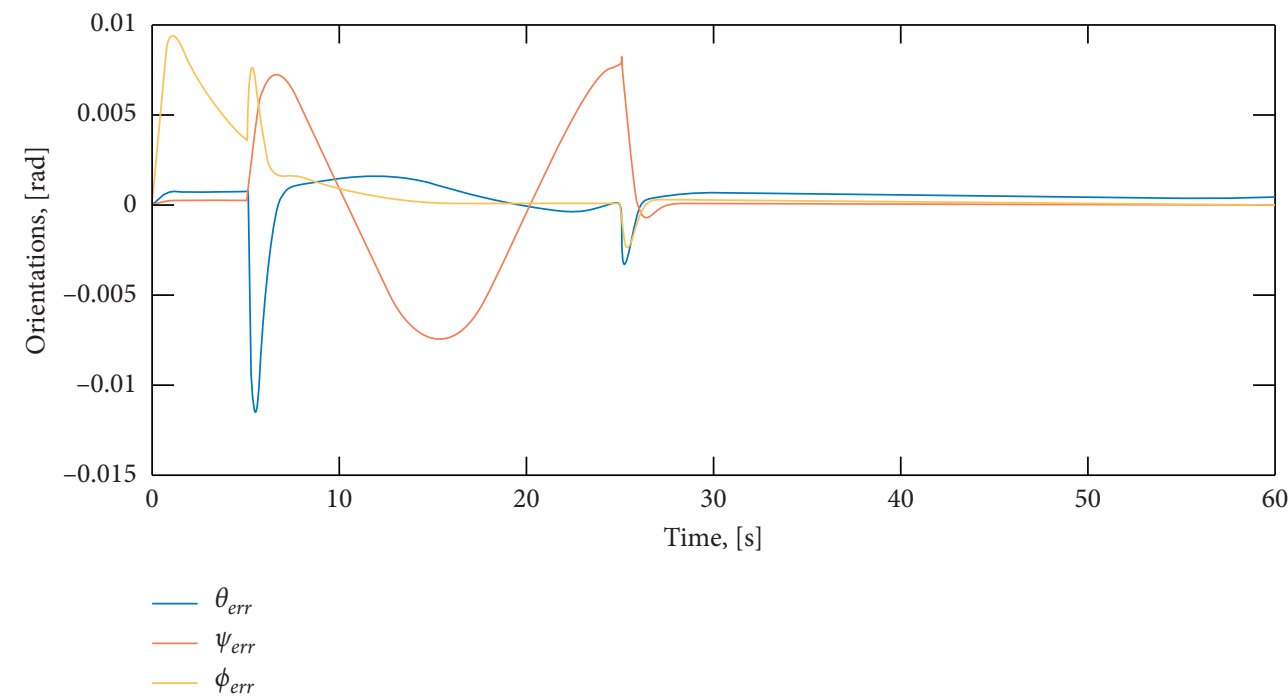

Figure 9: Orientation error curve in the free-flight experiment.

The expected flight trajectory of a given robot can be described as a circle. Analyze the experimental results and go through coordinate conversion. The flight trajectory is shown in Figure 8.
The results of this experiment are shown in Figures 9 and 10.

It can be seen from the figure that the posture of the robot can quickly stabilize after taking off to flying around 


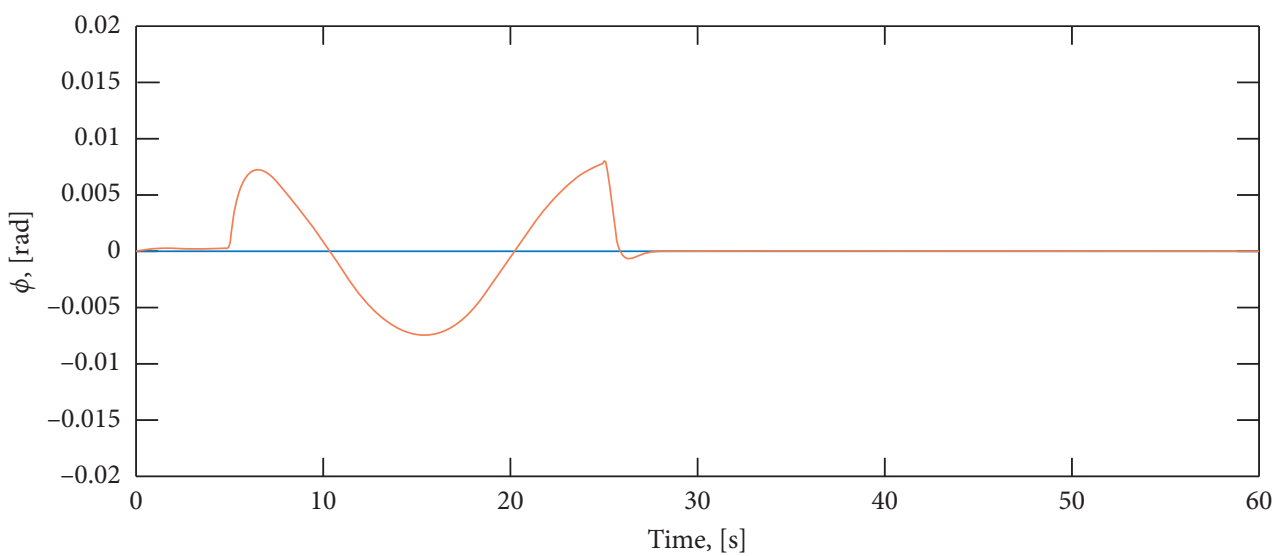

$\phi_{\text {des }}$

$\phi_{\text {est }}$

(a)

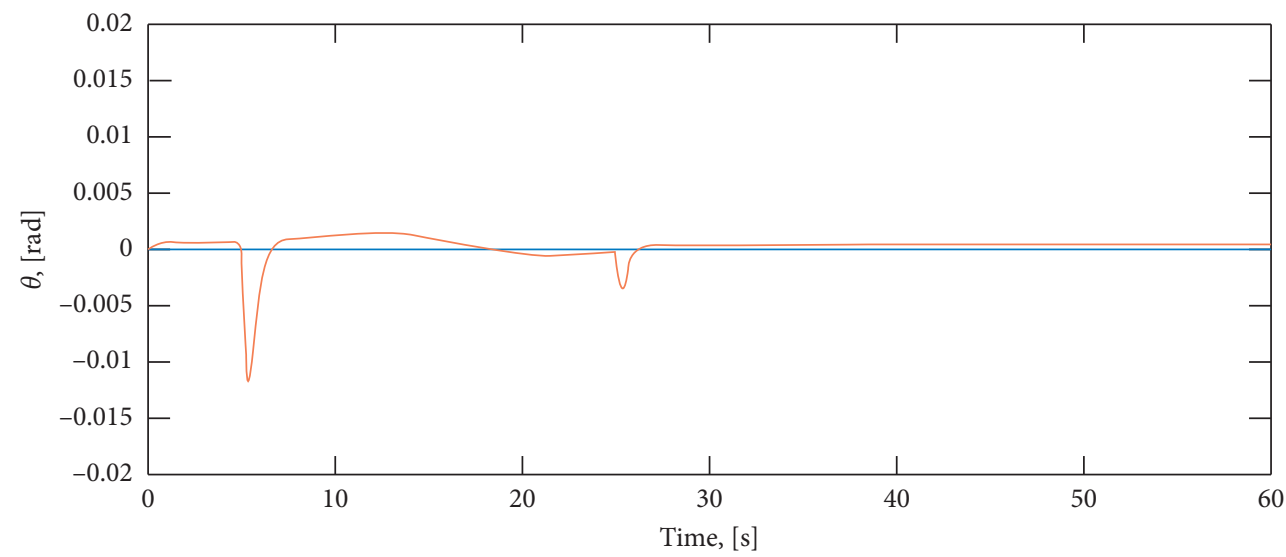

$\theta_{\text {des }}$

- $\theta_{\text {est }}$

(b)

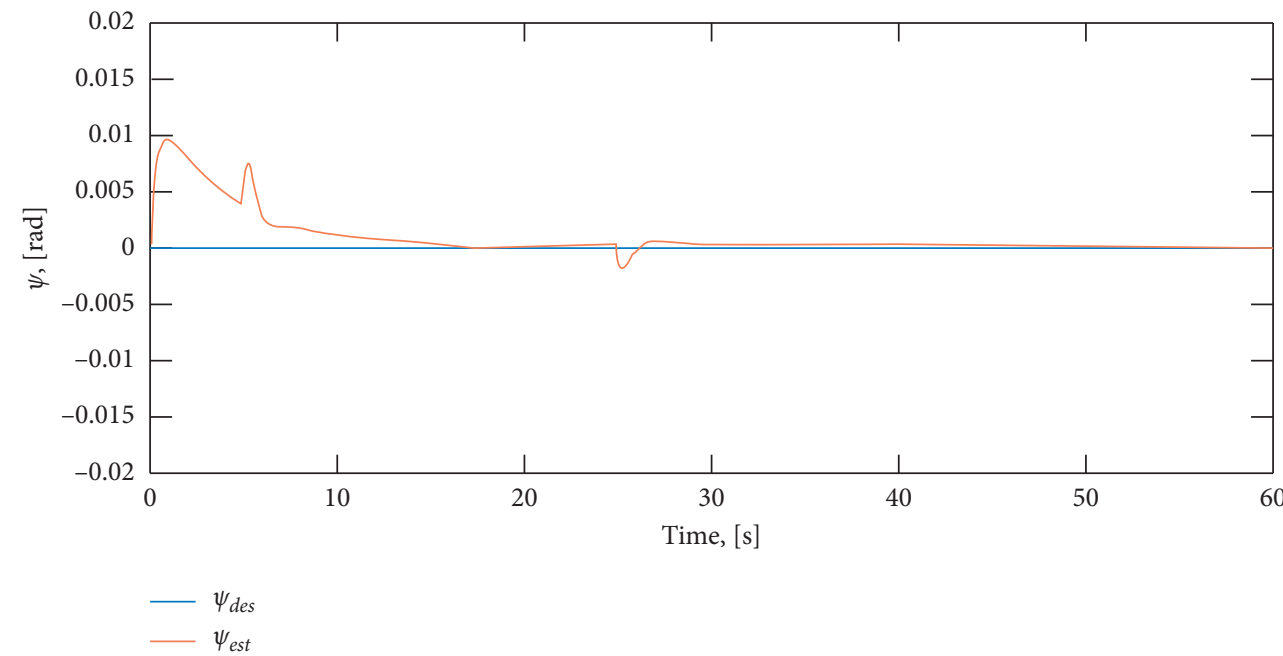

(c)

Figure 10: The (a) pitch angle, (b) roll angle, and (c) yaw angle in the free-flight experiment. 


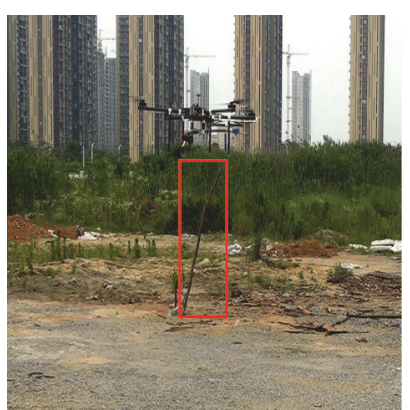

(a)

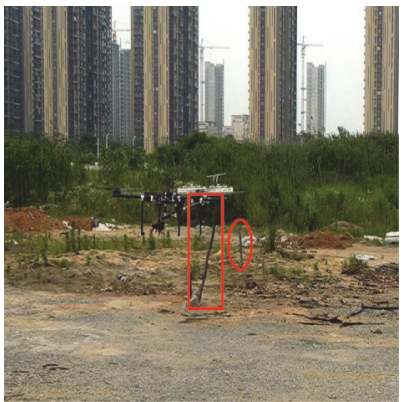

(d)

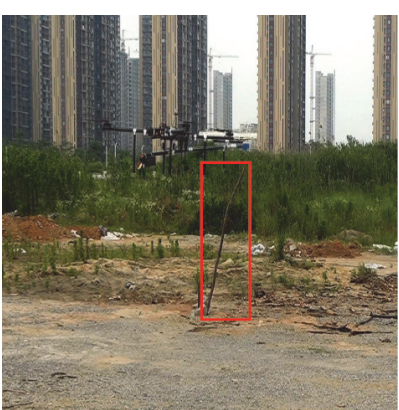

(b)

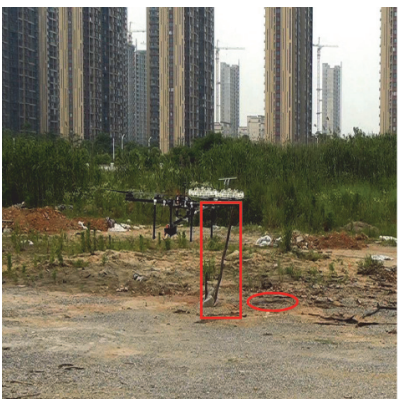

(e)

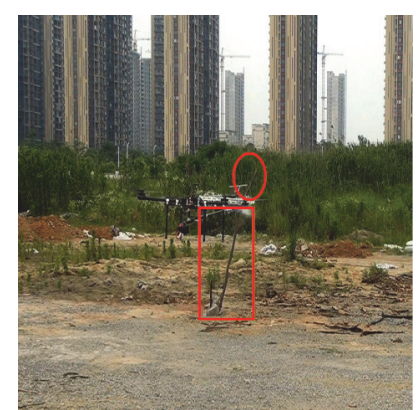

(c)

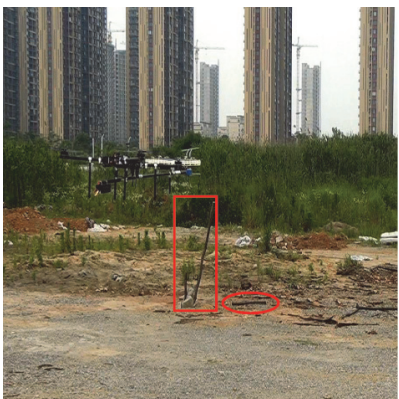

(f)

FIgURE 11: Snapshots of the outdoor tree cutting experiment.

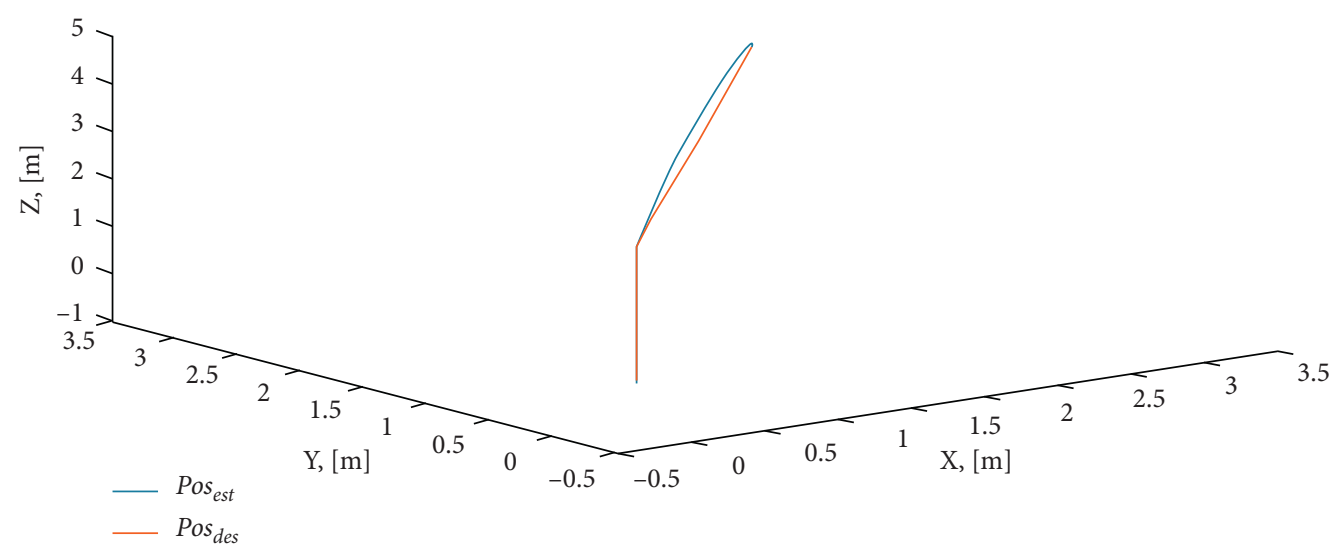

Figure 12: Tracking trajectory in the outdoor tree cutting experiment.

the trees. Figure 9 illustrates the change of attitude angle error in trajectory tracking. It can be seen that the error of the three angles does not change much and tends to stabilize quickly. Figure 10 shows the specific attitude angle change.

4.4. Outdoor Tree Cutting Experiment. This section mainly verifies the effectiveness of the designed controller during the APhI process. According to the results of modeling and analysis in Section 2, it can be seen that the primary disturbance in the process comes from the movement of the end effector and the change of the body's moment of inertia caused by contact with environmental objects.

We take the tree cutting experiment as an example. The basic idea of the experiment design is as follows: the whole process is divided into two parts. The first part shows the process of AMFCE approaching the target tree barrier after takeoff, during which the AMFCE is in free flight; the second part shows the process of AMFCE cutting the tree. During the process, the saw of the end effector was turned on and AMFCE switched from the free flight to cutting operation. When the body comes into contact with the tree, the AMFCE's moment of inertia matrix is changed, the control allocation matrix is switched, and the external disturbances are applied simultaneously.

The experiment is shown in Figure 11.

In Figure 11, the red box identifies the branches that need to be cut fixed on the ground. The red round frame is the part of the branch that fell after being cut. The change curve of position and posture is shown in Figures 12-14. 


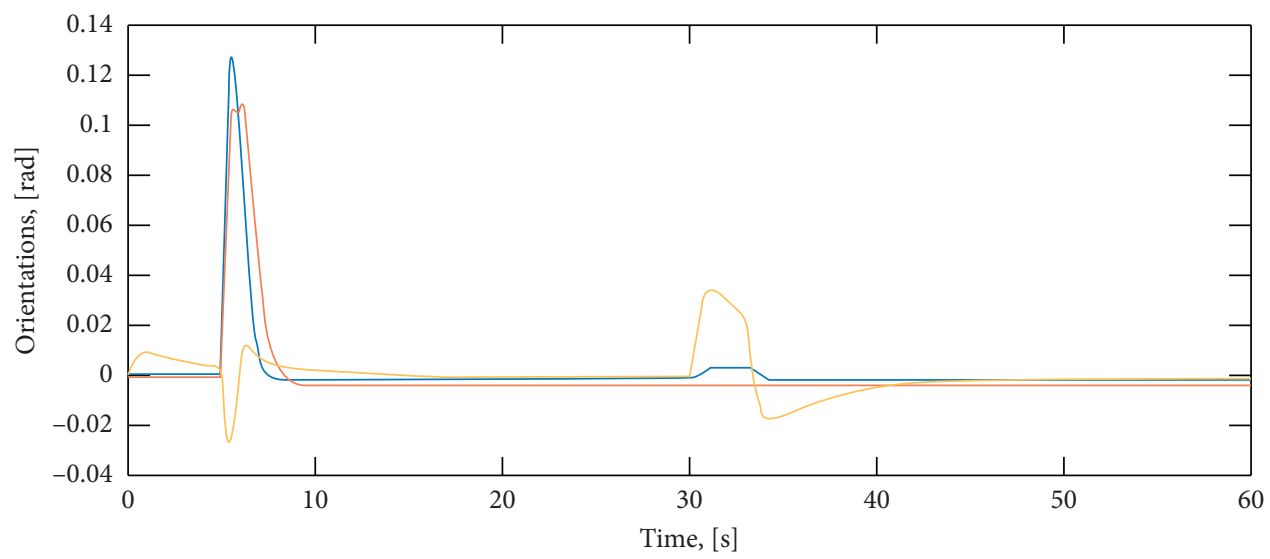

$-\theta_{\text {err }}$

$\psi_{\text {err }}$

$\phi_{\text {err }}$

Figure 13: Orientation error curve in the outdoor tree cutting experiment.

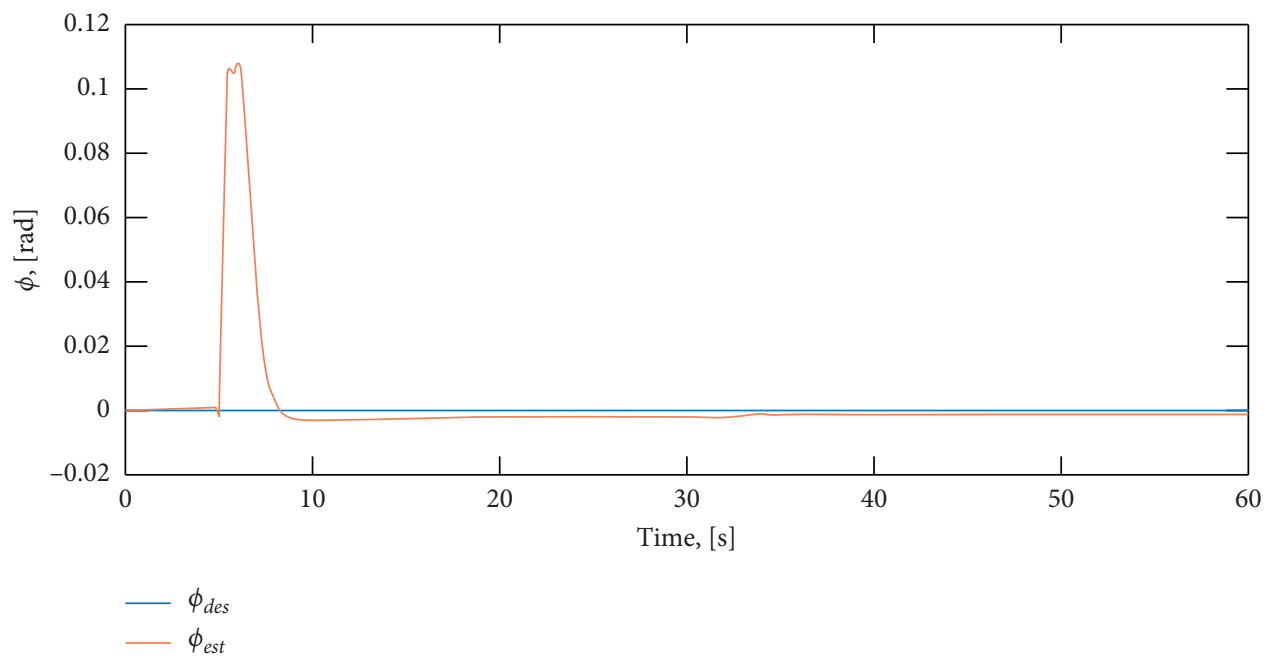

(a)

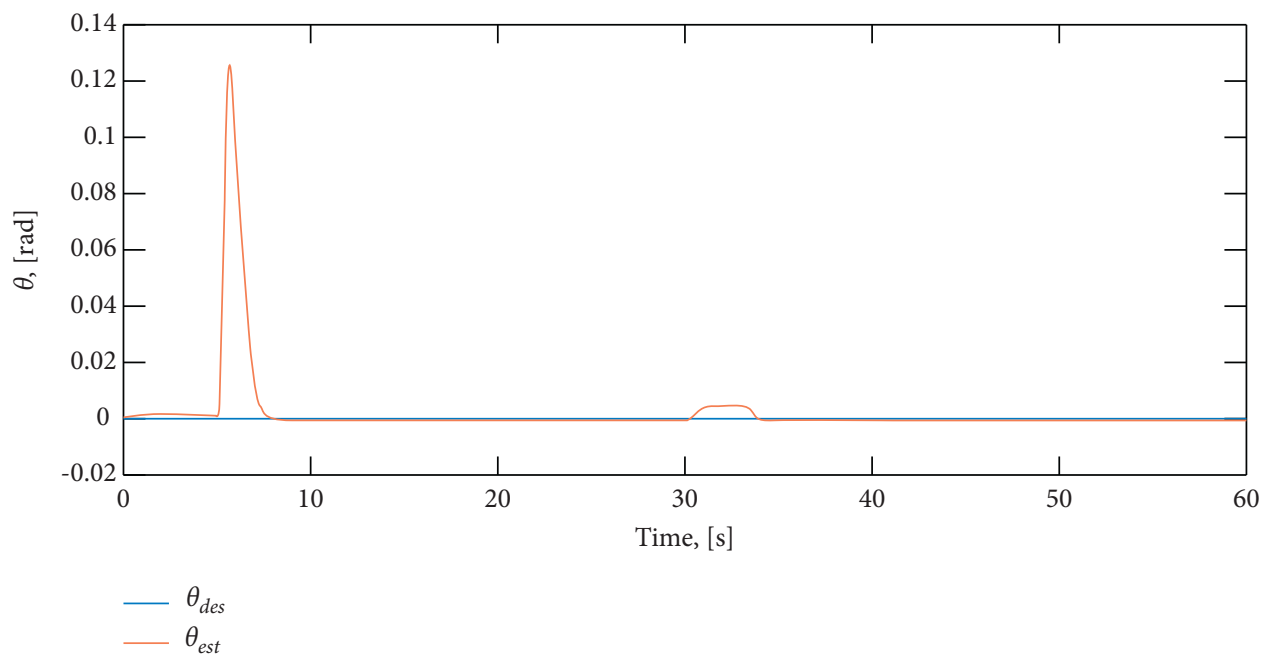

(b)

Figure 14: Continued. 


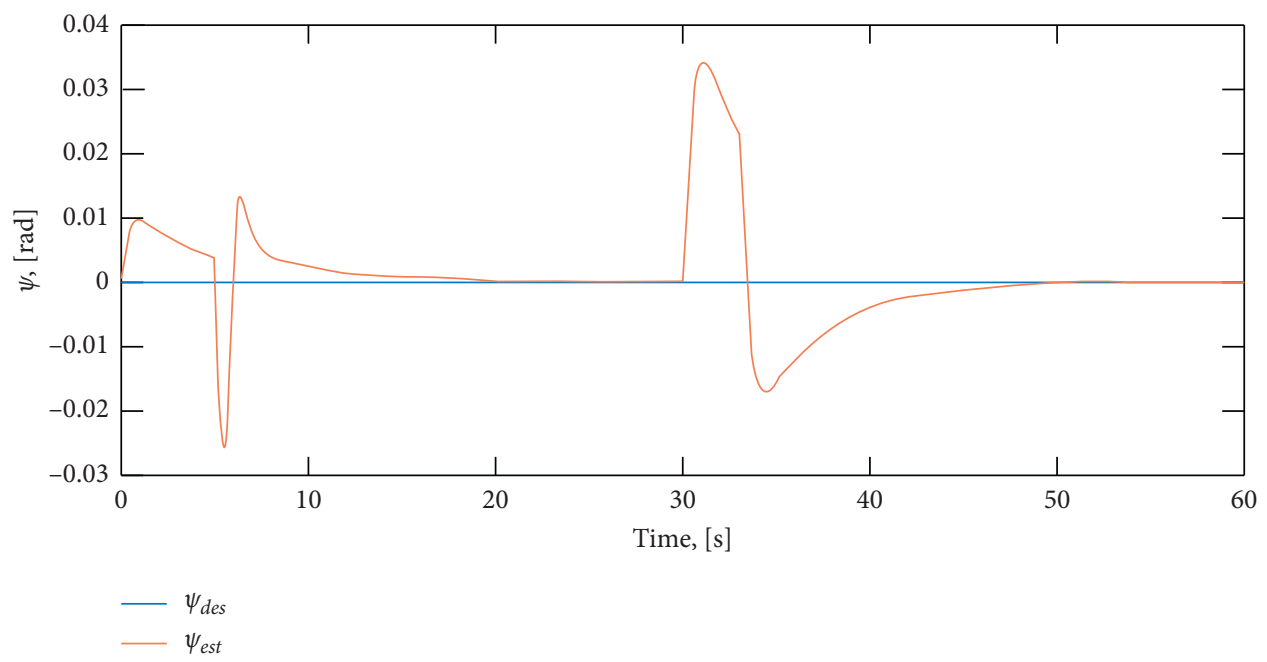

(c)

FIGURE 14: The (a) pitch angle, (b) roll angle, and (c) yaw angle in the outdoor tree cutting experiment.

As shown in Figures 13 and 14, the AMFCE is in free flight between 0 and $29 \mathrm{~s}$, the robot takes off and approaches the target tree. The controller adjusts the pitch angle of the body to make the AMFCE approach the target tree in the horizontal direction; between 30 and $34 \mathrm{~s}$, the AMFCE performs the cutting operation. During the cutting process, the body only changes slightly. After $34 \mathrm{~s}$, the robot completed the cutting and its posture and position quickly returned to normal.

\section{Discussion and Conclusions}

Throughout the development of the AMFCE, a range of issues were encountered on which we try to solve a problem of physical contact in the air. While accomplishing the aerial physical interaction has a range of advantages, it also has the following practical limitations:

(1) As it is difficult to maintain a tight fit, the wear of the parts will cause a significant backlash

(2) Unable to avoid overheating issues during use

(3) Frequent physical contact will produce a continuous force action on the inside and outside of the entire system, which may cause problems such as loose assembly and internal damage

(4) The thrust-to-weight ratio of the system is marginal, limiting its ability to carry payloads

In future research, we will continue to optimize the above limitations through design improvements. Overall, however, we demonstrated how an aerial manipulator with front cutting effector (AMFCE) addressed the aerial physical interaction (APhI) problem and realized cutting trees in the air with a saw. The experimental results show the effectiveness of our design. In conclusion, the AMFCE showed excellent capabilities and can push the boundaries of what is currently achievable by standard multirotors.

\section{Data Availability}

The figures, tables, and other data used to support this study are included within the article.

\section{Conflicts of Interest}

The authors declare that there are no conflicts of interest regarding the publication of this study.

\section{Acknowledgments}

This work was supported by the Guizhou Provincial Science and Technology Projects (Grant no. [2020]2Y044), the Science and Technology Projects of China Southern Power Grid Co. Ltd. (Grant no. 066600KK52170074), the Key Laboratory Projects of Aeronautical Science Foundation of China (Grant no. 201928052006), The Research Innovation Program for Postgraduates of Universities in Jiangsu Province (Grant no. KYLX16_0380), and the National Natural Science Foundation of China (Grant no. 61473144).

\section{References}

[1] H. Bonyan Khamseh, F. Janabi-Sharifi, and A. Abdessameud, "Aerial manipulation-a literature survey," Robotics and Autonomous Systems, vol. 107, pp. 221-235, 2018.

[2] F. Ruggiero, V. Lippiello, and A. Ollero, "Aerial manipulation: a literature review," IEEE Robotics and Automation Letters, vol. 3, no. 3, pp. 1957-1964, 2018.

[3] X. Meng, Y. He, J. Han, J. Han, and J. Han, "Survey on aerial manipulator: system, modeling, and control," Robotica, vol. 38, no. 7, pp. 1288-1317, 2020.

[4] M. Fanni and A. Khalifa, "A new 6-DOF quadrotor manipulation system: design, kinematics, dynamics, and control," IEEE, vol. 22, no. 3, pp. 1315-1326, 2017.

[5] P. Alexandre and D. Roque, Heterogeneous Collaborative Aerial Manipulation, KTH Royal Institute of Technology, Stockholm, Sweden, 2017. 
[6] R. Mahony, V. Kumar, and P. Corke, "Multirotor aerial vehicles: modeling, estimation, and control of quadrotor," IEEE Robotics and Automation Magazine, vol. 19, no. 3, pp. 20-32, 2012.

[7] D. Mellinger, M. Shomin, N. Michael, and V. Kumar, "Cooperative grasping and transport using multiple quadrotors," in Distributed Autonomous Robotic Systems, vol. 83, pp. 545-558, Springer Tracts in Advanced Robotics, Berlin, Germany, 2012.

[8] N. Michael, J. Fink, and V. Kumar, "Cooperative manipulation and transportation with aerial robots," Autonomous Robots, vol. 30, no. 1, pp. 73-86, 2011.

[9] J. Molina and S. Hirai, "Kinematic analysis of a novel skewgripper for aerial pruning tasks," in Proceedings of the $3 \mathrm{rd}$ International Conference on Mechatronics and Robotics Engineering, pp. 134-138, Paris, France, February 2017.

[10] J. Molina and S. Hirai, "Pruning tree-branches close to electrical power lines using a skew-gripper and a multirotor helicopter," in Proceedings of the 2017 IEEE/ASME International Conference on Advanced Intelligent Mechatronics, pp. 1123-1128, AIM, Munich, Germany, July 2017.

[11] J. Molina and S. Hirai, "Aerial pruning mechanism, initial real environment test," Robotics and Biomimetics, vol. 4, no. 1, pp. 127-132, 2017.

[12] N. Azami, P. Zarafshan, A. M. Kermani, M. Khashehchi, and S. Kouravand, "Design and analysis of an armed-octorotor to prune trees near the power lines," in Proceedings of the International Conference of Iranian Aerospace Society, Tehran, Iran, February 2017.

[13] H. Bolandi, M. Rezaei, R. Mohsenipour, H. Nemati, and S. M. Smailzadeh, "Attitude control of a quadrotor with optimized PID controller," Intelligent Control and Automation, vol. 4, no. 3, pp. 335-342, 2013.

[14] K. Nonami, F. Kendoul, S. Suzuki, W. Wang, and D. Nakazawa, Autonomous Flying Robots: Unmanned Aerial Vehicles and Micro Aerial Vehicles, Springer, New York, NY, USA, 2010.

[15] G. V. Raffo, M. G. Ortega, and F. R. Rubio, "Nonlinear $H \infty$ controller for the quad-rotor helicopter with input coupling," IFAC Proceedings Volumes, vol. 44, no. 1, 2011.

[16] C. Korpela, M. Orsag, M. Pekala, and P. Oh, "Dynamic stability of a mobile manipulating unmanned aerial vehicle," in Proceedings of the 2013 IEEE International Conference on Robotics and Automation, pp. 4922-4927, Karlsruhe, Germany, May 2013.

[17] Y. Liu, X. Huang, Y. Zhang, and Y. Zhou, "Dynamic stability and control of a manipulating unmanned aerial vehicle," International Journal of Aerospace Engineering, vol. 2018, Article ID 3481328, 13 pages, 2018.

[18] M. Kamel, K. Alexis, and R. Siegwart, "Design and modeling of dexterous aerial manipulator," in Proceedings of the IEEE International Conference on Intelligent Robots and Systems, pp. 4870-4876, Daejeon, South Korea, October 2016.

[19] B. Torchani, A. Sellami, and G. Garcia, "Sliding mode control of saturated systems with norm bounded uncertainty," in Proceedings of the 2012 16th IEEE Mediterranean Electrotechnical Conference, vol. 5, pp. 15-18, Yasmine Hammamet, Tunisia, March 2012.

[20] H. Karami and R. Ghasemi, "Fixed time terminal sliding mode trajectory tracking design for a class of nonlinear dynamical model of air cushion vehicle," SN Applied Sciences, vol. 2, no. 1, pp. 1-9, 2020.

[21] S. Bouabdallah and R. Siegwart, "Backstepping and slidingmode techniques applied to an indoor micro quadrotor," in
Proceedings of the IEEE International Conference on Robotics and Automation, pp. 2247-2252, Barcelona, Spain, April 2005.

[22] D. Kim, J. Di Carlo, B. Katz, G. Bledt, and S. Kim, "Highly dynamic quadruped locomotion via whole-body impulse control and model predictive control," 2019, http://arxiv.org/ abs/1909.06586.

[23] J. Zhang, X. Zhu, and Z. Zhou, "Design of time delayed control systems in UAV using model based predictive algorithm," in Proceedings of the 2010 2nd International Asia Conference on Informatics in Control, Automation and Robotics CAR 2010, vol. 1, pp. 269-272, Wuhan, China, March 2010.

[24] H. Xu, Z. Yang, L. Chang, K. Lu, C. Xu, and Q. Zhang, "ARSS: a novel aerial robot performs tree pruning tasks," Discrete Dynamics in Nature and Society, vol. 2020, Article ID 8883655, 14 pages, 2020.

[25] S. M. Chen Zengqiang and L. I. Yi, "ADRC-GPC control of a quad-rotor unmanned aerial vehicle," Journal of Harbin Institute of Technology, vol. 54, no. 3, pp. 955-962, 2014.

[26] M. Orsag, C. Korpela, and P. Oh, "Modeling and control of MM-UAV: mobile manipulating unmanned aerial vehicle," Journal of Intelligent and Robotic Systems, vol. 69, no. 1-4, pp. 227-240, 2013.

[27] I. Maza, K. Kondak, M. Bernard, and A. Ollero, "Multi-UAV cooperation and control for load transportation and deployment," Journal of Intelligent and Robotic Systems, vol. 57, no. 1-4, pp. 417-449, 2010.

[28] J.-B. Liu, J. Cao, A. Alofi, A. AL-Mazrooei, and A. Elaiw, "Applications of Laplacian spectra for n-prism networks," Neurocomputing, vol. 198, pp. 69-73, 2016.

[29] N. Koenig and A. Howard, "Design and use paradigms for gazebo, an open-source multi-robot simulator," in Proceedings of the 2004 IEEE/RSJ International Conference on Intelligent Robots and Systems (IROS) (IEEE Cat. No. 04CH37566), vol. 3, pp. 2149-2154, Sendai, Japan, September 2004.

[30] F. Furrer, M. Burri, M. Achtelik, and R. Siegwart, "RotorS-A modular gazebo MAV simulator framework," Studies in Computational Intelligence, Springer, New York, NY, USA, pp. 595-625, 2016. 